protocol in improving pain management in children attending the emergency department (ED). Pain is often suboptimally managed in children $^{1}$ despite evidence that quantification of pain severity is vital to enable provision of appropriate analgesia, and has been shown to reduce time to analgesia in previous studies. ${ }^{2}$ Prior to our audit, no protocol for paediatric pain assessment and management was in use in our ED.

We performed a retrospective assessment of 115 children aged $4-16$ years attending the ED with painful conditions such as trauma, abdominal pain, headache and head injury. Only $24 \%$ of children received analgesia at initial assessment, with a mean delay of $40 \mathrm{~min}$ (SE \pm 7.55$)$, and no child had a pain score performed. After introduction of a pain scoring chart (adapted from the Wong and Baker face chart $^{3}$ ), with a corresponding analgesia protocol, prospective assessment of a further 116 children (similar to the retrospective cohort in terms of age and diagnosis) showed that a pain score was more likely to be performed $(71 \%$ vs $0 \%$; $<0.001)$. More children were prescribed analgesia $(51 \%$ vs $24 \%$, $\mathrm{p}=0.001)$ and the mean delay to prescription was reduced to $15 \mathrm{~min}(\mathrm{SE} \pm 1.79 ; \mathrm{p}<0.001)$. When a pain score was performed, the drug choice was appropriate to pain score in $75 \%$ of cases, and, of these, when analgesia was given, dosage was appropriate in $93 \%$.

Introduction of this protocol therefore resulted in an increase in formal pain assessment and prescription of appropriate analgesia, and a reduction in time from triage to analgesia. The use of visual pain scoring analogue scales has a particular advantage in ethnically diverse populations such as that served by our ED, where English is a second language for many families, and is affordable and easily achievable. We would recommend introduction of these tools, and training in their use, to all personnel involved in management of paediatric patients with painful conditions. The introduction of such training, alongside appropriate protocols and scoring charts, can ensure that prescription of analgesia remains appropriate for paediatric patients, even within the setting of a general emergency department.

\section{Acknowledgements}

We thank Nicola Mayne, Paediatric Pharmacist, University College London Hospital and the Emergency Department staff, University College London Hospital for taking part in this study.
S Eisen, K Amiel

Paediatric and Adolescent Directorate, University College London Hospital, London, UK

Correspondence to: Sarah Eisen, 299B Upper Street, London N1 2TU, UK; saraheisen@hotmail.com

$$
\text { doi: } 10.1136 / \text { adc.2007.123372 }
$$

Financial support: None.

Competing interests: None.

\section{References}

1 Schechter NL. The undertreatment of pain in children: an overview. Pediatr Clin North Am 1989;36(4):781-94.

2 Somers L, Beckett MW, Sedgwick PM, et al. Improving the delivery of analgesia to children in pain. Emerg Med J 2001;18(3):159-61.

3 Whaley L, Wong DL. Nursing care of infants and children, 3rd edn. St Louis: CV Mosby, 1983.

\section{BOOK REVIEW}

\section{Health for all children, 4th edition}

Edited by David Hall, David Elliman. Oxford: Published by Oxford University Press, 2006, $£ 21.95$ (paperback), pp 422.

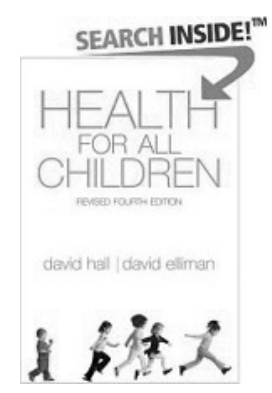

This 400-page volume has proved quite a difficult book to review. The 'Health for all children' books are a cornerstone of child health practice when considering broad populations of children. The publication of each edition is keenly awaited and goes on to influence both the organisation and prosecution of child health services throughout the UK and beyond. This revision of the fourth edition is due to the publication of several important documents concerning the health and welfare of children in the UK, namely the National service framework for children and Every child matters. Emerging data from SureStart programmes are also starting to shape the future direction of health and surveillance services for children.

The book follows the familiar layout of previous publications, whereby the opening chapters set out the fundamental concepts underlying preventive child health programmes and summarises the evidence for child health promotion in its widest sense (promoting optimal child development and promoting/supporting parenting) before later chapters explore more specific areas of screening (primary and secondary)-for example, in respect of iron deficiency anaemia and hearing deficits. The revised edition benefits from modern, up-to-date formatting and is easier to read than the original.

Those who have read previous editions of the book will be aware that large working groups contribute to each chapter. However in this revised edition, Hall and Elliman have taken the task of updating the various chapters on themselves. The end result is a book which although looking updated, feels very much like the original fourth edition. In fact, astute and keen followers of the H4AC website will recognise that the revised components of the book are available online, so the revised edition avoids them having to cut and paste this information themselves. Unfortunately, this has led to some pagination issues-for example, the executive summary recommends a screening programme said to be detailed on page 351 of the book. However, the table is on page 351 of the original fourth edition and page 341 of the revised edition. These irritations should not detract from an appreciation of what is an immense work of scholarship that in a concise volume appraises a wide variety of child and family focused interventions from a scientific, sociological and probably a political perspective.

I think that many paediatricians involved with child public health, community-based work and child health promotion will already have the fourth edition, and so the question is obviously whether purchase of this revised edition is warranted. For those trainees and other first-time buyers wishing to gain an insight into these aspects of child public health this book will be invaluable and I recommend it without hesitation. It is also highly recommended for those aficionados who have the original fourth edition but do not have access to the Health for all children website. However, for those who remain up to date with these issues the decision to upgrade to this revised edition lies in equipoise. The book's reasonable price may well tip the balance.

It remains my enduring hope that the increased accessibility of the revised edition will tempt those in positions of influence in primary care organisations and local authorities to read it and to commit to adequately funding the implementation of its sensible, soundly researched and well-reasoned recommendations.

Adrian Brooke

\title{
Call for Book Reviewers
}

Book reviews are a popular feature of $A D C$, and many readers use them to decide how best to spend scarce library funds. We need to recruit a team of willing reviewers, both generalists and specialists, who are prepared to read and review new books (and CD-ROMs, etc) within a three-month deadline: could you help? You will have the option to decline if you can't manage a review in time.

Unfortunately $A D C$ cannot pay reviewers, but you will be able to keep the book for yourself or your department. Trainees are particularly welcome to apply.

For logistical reasons reviewers should be based in the UK or Republic of Ireland and internet access is essential.

Please contact archdischild@bmigroup.com with brief details of special interests and reviewing experience, if any (include BOOK REVIEWS in the subject field). 